\title{
Using Ionic Strength to Control Liquid-Ordered/Liquid-Disordered Separation
}

\author{
Kalani J. Seu, Emily R. Lamberson and Jennifer S. Hovis* \\ Department of Chemistry, Purdue University, West Lafayette, IN 47907-2018
}

\section{Supporting Information}

\section{Experimental Methods}

Materials: 1,2-dioleoyl-sn-glycero-3-phosphocholine (DOPC), 1,2-dipalmitoyl-sn-glycero-3phosphocholine (DPPC), 1,2-distearoyl-sn-glycero-3-phosphocholine (DSPC), cholesterol, and 1-palmitoyl-2-[6-[(7-nitro-2-1,3-benzoxadiazol-4-yl)amino]hexanoyl]-sn-glycero-3phosphocholine (NBD-PC) were purchased from Avanti Polar Lipids. $N$-(Texas Red sulfonyl)1,2-dihexadecanoyl-sn-glycero-3-phosphoethanolamine (TR-DHPE) and CoverWell ${ }^{\mathrm{TM}}$ perfusion $^{-}$ chamber gaskets were purchased from Invitrogen-Molecular Probes, Inc. Glass coverslips, $22 \times$ 30 \#1.5 were purchased from Fisher Scientific. ICN 7X detergent was purchased from ICN.

Vesicle supported lipid bilayer preparation: Appropriate mixtures of lipids and cholesterol in chloroform were dried under $\mathrm{N}_{2}(\mathrm{~g})$ and placed under vacuum for $1 \mathrm{~h}$. Large unilamellar vesicles (LUVs) were formed by extruding the rehydrated lipid suspension through a polycarbonate membrane with $50 \mathrm{~nm}$ pores a minimum of 21 times. Solid glass supports were detergent cleaned by washing in a dilute solution of ICN 7X detergent for at least 10 minutes, rinsed excessively in $18 \mathrm{M} \Omega-\mathrm{cm}$ water, and dried under a stream of $\mathrm{N}_{2}(\mathrm{~g})$ prior to any additional surface treatment. Piranha etched glass slides were exposed to a solution of 4:1 (v/v) concentrated sulfuric acid $\left(\mathrm{H}_{2} \mathrm{SO}_{4}\right)$ and $30 \%$ hydrogen peroxide $\left(\mathrm{H}_{2} \mathrm{O}_{2}\right)$, rinsed excessively in 18 $\mathrm{M} \Omega-\mathrm{cm}$ water and dried under a stream of $\mathrm{N}_{2}(\mathrm{~g})$. The slides were used within a few minutes after treatment. Bilayers were formed by vesicle fusion on the treated slides.

\section{Supplemental figures}

Domain movement: As mentioned in the text, the flat liquid-ordered domains were occasionally observed to move and merge. An example is shown below.

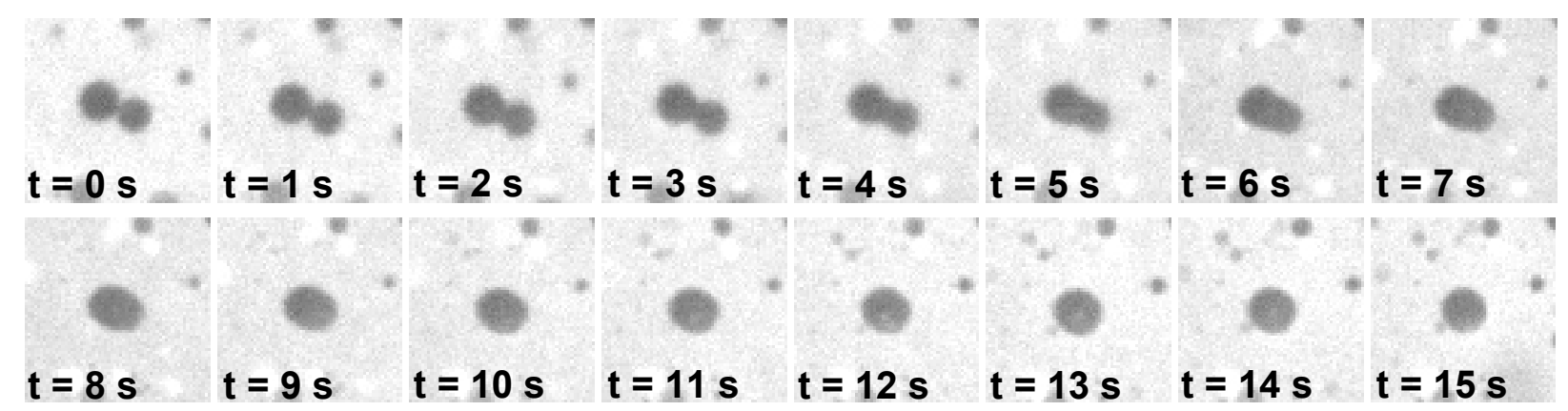

Figure S1: An example of two domains coalescing. The bilayer composition is 1:1 DOPC:DPPC, $30 \%$ cholesterol, $0.5 \%$ Texas Red, and the bulk solution is water. Images were collected using a 100x oil-immersion objective and are $10 \mu \mathrm{m} \times 10 \mu \mathrm{m}$. 
Method 2: As mentioned in the text if the bilayers formed by method 2 are subjected to repeated cycling of the ionic strength domains often appear.



Figure S2: A bilayer formed using method 2 does not show domains after completion of the method (left); however, when the bilayer was incubated an additional 15 minutes in $500 \mathrm{mM}$ $\mathrm{NaCl}$ and rinsed in water, domains were observed. Images were taken using a 40x air objective, and the images are $40 \mu \mathrm{m} \times 40 \mu \mathrm{m}$. 